\title{
The Casimir free energy in high- and low-temperature limits
}

\author{
V B Svetovoy ${ }^{1}$ and R Esquivel ${ }^{2}$ \\ ${ }^{1}$ MESA+ Research Institute, University of Twente, PO 217, 7500 AE Enschede, The Netherlands \\ 2 Instituto de Fisica, Universidad Nacional Autónoma de México, Apartado Postal 20-364, \\ DF 01000 México, Mexico \\ E-mail: V.B.Svetovoy@el.utwente.nl and raul@fisica.unam.mx
}

Received 3 November 2005, in final form 3 February 2006

Published 10 May 2006

Online at stacks.iop.org/JPhysA/39/6777

\begin{abstract}
The problem with the temperature dependence of the Casimir force is investigated. We analyse high-temperature limit analytically making calculations at real frequencies. The purpose is to answer the question why there is no continuous transition between real and ideal metals and why the result does not depend on the relaxation frequency. It is found that the contribution of evanescent s polarized fields is finite even for an infinitely small relaxation frequency (plasma model) and exactly cancels the contribution of propagating fields. For the ideal metal the evanescent fields do not contribute at all. The lowtemperature limit is analysed to establish behaviour of the entropy at $T \rightarrow 0$. It is stressed that the nonlocal effects are important in this limit because the mean free path for electrons becomes larger than the field penetration depth. In this limit $v_{F} / a$ plays the role of the relaxation frequency, where $v_{F}$ is the Fermi velocity and $a$ is the distance between plates. It is indicated that the Leontovich approximate impedance cannot be used for calculations because it is good for the description of propagating but not evanescent fields. It is found that due to nonlocality the Casimir entropy approaches zero at $T \rightarrow 0$ when s polarization does not contribute to the classical part of the Casimir force.
\end{abstract}

PACS numbers: $12.20 . \mathrm{Ds}, 42.50 . \mathrm{Lc}, 3.70 .+\mathrm{k}$

\section{Introduction}

An attractive force between uncharged metallic plates, predicted in 1948 by Casimir [1], is one of the most striking macroscopic manifestations of quantum vacuum [2, 3]. Recently this 
force became a subject of systematic experimental investigation [4-11]. The force between ideal metals at zero temperature [1],

$$
F=-\frac{\pi^{2} \hbar c}{240 a^{4}},
$$

depends only on the separation $a$ and fundamental constants. In reality the force is measured at a finite temperature between deposited metallic films, which have finite conductivity and roughness. Correction to equation (1) due to a finite conductivity can be as large as $50 \%$ for small separations $a \sim 100 \mathrm{~nm}$. Contribution of the finite temperature to this correction is not large but caused a lot of controversy in the literature (see [12] for a recent review). The essence of the problem lies in the classic contribution to the Casimir force, which dominates at large distances between plates or at a high-temperature. Calculations made for ideal metals at a finite temperature $[13,14]$ showed that $\mathrm{s}$ and $\mathrm{p}$ polarized modes of electromagnetic field gave equal contributions to the force. At the same time the Lifshitz theory of fluctuating fields $[15,16]$ predicted zero contribution for s polarization. The problem was recognized for the first time many years ago. For reconciliation of the results Schwinger, DeRaad, and Milton (SDM) [17] proposed a special prescription to be used with the Lifshitz formula: one must take first the limit $\varepsilon \rightarrow \infty$ for the metal permittivity and only then allow the frequency $\omega$ to go to zero. Modern calculations concerned with nonideal metals were confronted with the problem again.

Different approaches to resolve the problem have been proposed in the literature, which resulted in different temperature corrections to the Casimir force. Boström and Sernelius [18] used the Lifshitz formula with the Drude dielectric function (4) and found that s polarization did not contribute. In this approach there is no continuous transition to the ideal metal case and the predicted temperature correction is in contradiction with the Lamoreaux experiment [4]. However, physically this approach is well motivated. Bordag et al [19] used the plasma model dielectric function, for which the dielectric function $\varepsilon$ at low frequencies increases faster $\left(\omega^{-2}\right)$ than for the Drude model $\left(\omega^{-1}\right)$. They found that s polarization gives finite contribution in the classical limit, which coincides with the ideal metal result when the plasma frequency $\omega_{p}$ is going to infinity. The temperature correction in this approach is very close to that for the ideal metal and negligible at small separations between plates. A weak point of this approach is that no known material behaves at a low frequency according to the plasma model. Svetovoy and Lokhanin [20] proposed to use SDM prescription for the $n=0$ term in the Lifshitz formula for real metals also. Later it was shown [21] that this prescription follows from a very general dimensional analysis of the classical contribution to the force if one demands continuous transition to the ideal metal case. The temperature correction happened to be small but observable at small separations between bodies. A reasonable objection [22] against the last approach was that maybe we should not demand a continuous transition to the ideal metal because it does not exist. However, one has to be cautious here because absence of the continuous transition is an exceptional physical situation.

According to the Nernst heat theorem the entropy has to go to zero in the limit of zero temperature. It was noted [23] that the Drude relaxation frequency $\omega_{\tau}$ vanishes with $T$ and, therefore, the plasma dielectric function is realized at $T \rightarrow 0$. In this case the leading term in the temperature correction is $\sim T^{3}[19]$ and the entropy is safely going to zero as $S \sim T^{2}$. Two other approaches predict the leading term in the correction $\sim T$ and finite entropy at $T=0$, positive and negative for the approaches [20] and [18], respectively. However, the following analysis revealed that the situation is not as simple. It was indicated [22, 24] that any real material contains a number of defects, which are responsible for the residual resistivity at $T=0$. Equivalently it means that $\omega_{\tau}$ becomes very small but finite at $T=0$. In this case, the entropy disappears at a sufficiently low-temperature $[22,24]$. 
We would like to emphasize that at low-temperatures the anomalous skin effect plays an important role [25] and must be taken into account in any reasonable calculations. Because $\omega_{\tau}$ decreases fast with the temperature, at a sufficiently low-temperature inevitably the mean free path $l=v_{F} / \omega_{\tau}(T)$ for electrons becomes much larger than the field penetration depth $\delta$. When this happens the relaxation frequency no longer plays a physical role. Physical significance gets the other frequency $v_{F} k$, where $k$ is the wave number. Appropriate procedures taking into account nonlocal effects in the calculation of the Casimir force were developed independently in two recent studies [26, 27].

\section{The force in the high-temperature limit}

The Casimir force at nonzero temperature between plates made of real materials is given by the Lifshitz formula [16]. For the free energy $\mathcal{F}(a, T)$ this formula can be presented in the following form,

$$
\mathcal{F}(a, T)=\frac{k T}{2 \pi} \sum_{n=0}^{\infty}, \int_{0}^{\infty} \mathrm{d} q q\left[\ln \left(1-r_{s}^{2} \mathrm{e}^{-2 k_{0} a}\right)+\left(r_{s} \rightarrow r_{p}\right)\right],
$$

where the sum is running over the Matsubara frequencies $\zeta_{n}=2 \pi k T n / \hbar, k_{0}=\sqrt{\zeta_{n}^{2} / c^{2}+q^{2}}$, and $q$ is the momentum along the plates. In equation (2) $r_{s}$ and $r_{p}$ are the Fresnel reflection coefficients depending on the material dielectric function $\varepsilon=\varepsilon(i \zeta)$ at imaginary frequencies.

The problem with the thermal correction comes from the $n=0$ term in equation (2), which will be denoted as $\mathcal{F}_{0}(a, T)$. For the ideal metal $r_{s}=r_{p}=1$ and both polarizations contribute equally to the free energy:

$$
\mathcal{F}_{0}(a, T)=-\frac{k T}{16 \pi a^{2}} \zeta(3)(1+1),
$$

where units explicitly show equal contributions from $\mathrm{s}$ and $\mathrm{p}$ polarizations. Real metals can be described at low frequencies by the Drude dielectric function

$$
\varepsilon(\mathrm{i} \zeta)=1+\frac{\omega_{p}^{2}}{\zeta\left(\zeta+\omega_{\tau}\right)}
$$

where the parameters $\omega_{p}$ and $\omega_{\tau}$ are the plasma and relaxation frequencies for a given metal respectively. This formula is working especially well in the zero-frequency limit $\zeta \ll \omega_{\tau}$ where it expresses Ohm's law. Using function (4) we can see that $r_{s}=0$ and $r_{p}=1$. In this case s polarization does not contribute to $\mathcal{F}_{0}(a, T)$. Note that this conclusion does not depend on the material parameters. Thus, there is no continuous transition between real and ideal metals that is puzzling.

The Lifshitz formula in the form (2) does not tell us too much about the origin of the problem since it is written for imaginary frequencies and significant physical information is lost. Here we would like to analyse the classical contribution to the Casimir free energy at real frequencies. Our approach is analytical but some work in this direction was already done using numerical calculations [28].

The classical contribution to the free energy written as the integral over real frequencies $\omega$ and momenta along the plates $q$ is

$$
\mathcal{F}_{0}(a, T)=\frac{k T}{2 \pi^{2}} \operatorname{Im} \int_{0}^{\infty} \frac{\mathrm{d} \omega}{\omega} \int_{0}^{\infty} \mathrm{d} q q\left\{\ln \left(1-r_{s}^{2} \mathrm{e}^{2 \mathrm{i} k_{0} a}\right)+\left(r_{s} \rightarrow r_{p}\right)\right\},
$$

where $k_{0}=\sqrt{\omega^{2} / c^{2}-q^{2}}$. In the classical limit we changed $\operatorname{coth}(\hbar \omega / 2 k T) \rightarrow 2 k T / \hbar \omega$. We would like to understand why the classical term does not depend on the parameters of the metal 


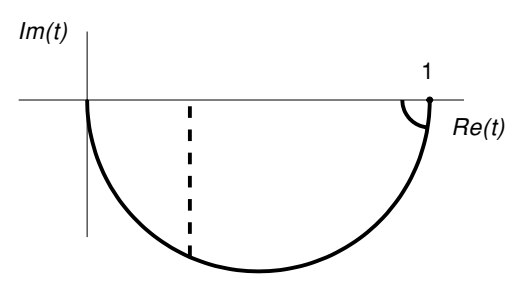

Figure 1. The integration contour in the complex plane $t$.

and why s polarization does not contribute. The Drude function (4) is used for calculations. This function is working especially well at low frequencies, which are the main range of our concern. As the source of controversy only s polarization will be discussed. To make calculations at real frequencies one has to separate contributions of propagating, $\omega / c>q$, and evanescent, $\omega / c<q$, fields.

Let us consider first the evanescent fields. Introducing a new variable

$$
x=2 a \sqrt{q^{2}-\omega^{2} / c^{2}}
$$

the contribution of s polarization can be written as

$$
\mathcal{F}_{0}^{s, \text { ev }}=\frac{k T}{8 \pi^{2} a^{2}} \operatorname{Im} \int_{0}^{\infty} \frac{\mathrm{d} \omega}{\omega} \int_{0}^{\infty} \mathrm{d} x x \ln \left(1-r_{s}^{2} \mathrm{e}^{-x}\right) .
$$

With the Drude dielectric function the reflection coefficient will be

$$
r_{s}=\frac{x-\sqrt{x^{2}+R^{2} \omega /\left(\omega+\mathrm{i} \omega_{\tau}\right)}}{x+\sqrt{x^{2}+R^{2} \omega /\left(\omega+\mathrm{i} \omega_{\tau}\right)}}, \quad R=\frac{\omega_{p}}{\omega_{c}}, \quad \omega_{c}=\frac{c}{2 a},
$$

where $\omega_{c}$ is very small in the range of interest (classical limit). For this reason the ratio $R$ is very large. In the case of ideal metal, $r_{s}=1$, the evanescent contribution to the free energy is zero because the integrand in (7) is real.

It seems that one can neglect $x^{2} \sim 1$ in comparison with large $R^{2}$ in equation (8) but this is not true. Even very small relaxation frequency $\omega_{\tau}$ plays an important role in integral (7). The smaller $\omega_{\tau}$ the lower frequencies give significant contribution to the integral but the contribution itself does not depend on $\omega_{\tau}$. The only thing which matters is that $\omega_{\tau}$ exists. It becomes obvious if one introduces the dimensionless frequency $\Omega=\omega / \omega_{\tau}$. After this substitute the integral does not depend on $\omega_{\tau}$ at all. Note that this is true only in the classical limit. To calculate the integral we integrate first over frequencies and for that introduce a new complex variable

$$
t=\frac{\omega}{\omega+\mathrm{i} \omega_{\tau}}=\frac{\Omega}{\Omega+\mathrm{i}} .
$$

After that the integral over $\omega$ becomes

$$
I(x)=\operatorname{Im} \int \frac{\mathrm{d} t}{t(1-t)} \ln \left(1-r_{s}^{2}(t, x) \mathrm{e}^{-x}\right)
$$

In the complex plane $t$ the integral is running over the half of the circle (see figure 1) from $t=0$ to $t=1$. The pole at $t=0$ is actually absent since $r_{s}(0, x)=0$. Closing the contour with the real axis one finds that the integral is reduced to the residue at the pole $t=1$. This is because the integrand is a real function at real $t$. Since $R$ is large the reflection coefficient at $t=1$ with a high precision is $r_{s}(1, x)=1$. Integrating finally over $x$ one finds

$$
\mathcal{F}_{0}^{s, \mathrm{ev}}=\frac{k T}{16 \pi a^{2}} \zeta(3) .
$$


This way of calculations, however, does not reveal information on the frequencies which contribute significantly to the free energy. To find these frequencies one can close the contour earlier as shown in figure 1 with the dashed line. It is equivalent to the upper limit cutoff $\omega_{0}$ in integration over $\omega$. The result will be the same as (11) while $r_{s}\left(t_{0}, x\right) \simeq 1$, where $t_{0}$ is connected with the cutoff frequency by equation (9). The integral becomes sensitive to the value of the cutoff frequency only at

$$
\omega_{0}=\omega_{\tau}\left(\frac{\omega_{c}}{\omega_{p}}\right)^{2}
$$

which can be considered as a frequency giving the most important contribution to the free energy. This frequency is extremely small in the classical limit. This fact was already discovered by Torgerson and Lamoreaux [28] in numerical calculations.

The case of propagating fields is more complicated technically but the idea is essentially the same. We omit technical details and present here only the final result:

$$
\mathcal{F}_{0}^{s, p r}=-\frac{k T}{16 \pi a^{2}} \zeta(3)
$$

There is no need of special analysis of important frequencies since the propagating fields have resonance character and these frequencies are $\omega \sim \omega_{c}$.

It is clear that absence of the continuous transition between real and ideal metals happens due to evanescent fields. The contribution of propagating fields (13) coincides with that for the ideal metal (3). The evanescent fields cancel it precisely. The exact value of the relaxation frequency is absolutely not important. Even an infinitely small relaxation frequency would give the same result (11). In this sense the correct use of the plasma model would give the same result as the Drude model. We cannot keep the relaxation frequency in the plasma model precisely zero because the dispersion relations will be broken, but $\omega_{\tau}$ can be arbitrarily small. In all physical limiting cases the transition to zero frequency must be the last.

\section{Entropy in the low-temperature limit}

As was explained in the introduction at low-temperatures the nonlocal effects become important. Nonlocal interaction between a material and an electromagnetic field is described by two material dielectric functions $\varepsilon_{t}(k, \omega)$ and $\varepsilon_{l}(k, \omega)$. They give response of the material to transverse ( $t$ ) or longitudinal (l) electric field. For metals these functions can be found, for example, by solving the Boltzmann kinetic equation [29]. An important parameter characterizing the nonlocality at imaginary frequencies, $\omega=\mathrm{i} \zeta$, is

$$
v=v_{F} \frac{k}{\zeta+\omega_{\tau}}
$$

The range of the anomalous skin effect corresponds to large values of $v$. When the Casimir force is calculated, $k=\sqrt{k_{z}^{2}+q^{2}}$ is restricted by the condition $k \geqslant q \sim 1 / 2 a$. On the other hand, the denominator in equation (14) is small and the condition $v \gg 1$ will be fulfilled at a sufficiently low frequency (temperature). In this limit the dielectric functions behave as [30]

$$
\varepsilon_{l}(\zeta, k)=1+3\left(\frac{\omega_{p}}{v_{F} k}\right)^{2}, \quad \varepsilon_{t}(\zeta, k)=1+\frac{3 \pi}{4} \frac{\omega_{p}^{2}}{\zeta v_{F} k} .
$$

One can immediately see that the relaxation frequency falls out from the dielectric functions. The longitudinal function, $\varepsilon_{l}$, does not depend on frequency at all; its $k$ dependence describes the Thomas-Fermi screening of the longitudinal electric field. In the transverse function, $\varepsilon_{t}$, the product $v_{F} k$ plays a role of the relaxation frequency. 
Reflection properties of metals can be described with the surface impedances $Z_{s}(\omega, q)$ and $Z_{p}(\omega, q)$ [31,32], which can be expressed via the nonlocal dielectric functions. For the anomalous skin effect these impedances were found in [30] (see also [27]). When $q \rightarrow 0$ (propagating field) both impedances coincide and reproduce the Leontovich impedance for the anomalous skin effect [33]. However, for the Casimir problem $q \sim 1 / 2 a$ is always finite and at some low-temperature the Leontovich approximation will fail. This temperature is defined by the condition

$$
k T \ll \frac{\hbar \omega_{c}}{2 \pi} \frac{v_{F}}{c},
$$

which follows from the condition of nonlocality $v \gg 1$. In the low-temperature limit the impedances are [27, 30]

$$
Z_{s}=\frac{\zeta}{c q}, \quad Z_{p}=\frac{q^{2}}{\sqrt{3}} \frac{c v_{F}}{\zeta \omega_{p}} .
$$

These impedances differ from the Leontovich impedance that indicates significant influence of the evanescent fields. For this reason the results based on the Leontovich approximation $[25,34]$ must be reconsidered. The impedance $Z_{s}$ at a low-temperature approaches its local limit, for which s polarization does not contribute to the $n=0$ term in (2). In contrast with the high-temperature (large-distance) limit the $n \neq 0$ terms are also important and they cancel linear in $T$ term in the free energy at $T \rightarrow 0$. Calculations made in [27] gave for the temperature-dependent part of the free energy

$$
\Delta \mathcal{F}=\frac{k T A^{2}}{8 \pi a^{2}}(0.0146-0.0041 A), \quad A=\left(\frac{3 \pi^{2}}{2} \frac{c}{v_{F}} \frac{\omega_{p}^{2}}{\omega_{c}^{2}} \frac{k T}{\hbar \omega_{c}}\right)^{1 / 3} \ll 1
$$

This asymptotic behaviour is realized at temperatures much smaller than the limit (16). In contrast with [27] we presented here also the next-to-leading term in the expansion in $A$. This term is important because the leading one does not depend on distance between bodies $a$ and does not contribute to the force. For this reason, it has no relation to the Casimir free energy and can be omitted. This moment was missed in [27].

At very low-temperatures the free energy behaves as $T^{2}$ and the entropy $S=-\partial \Delta \mathcal{F} / \partial T$ is going to zero at $T \rightarrow 0$ as $T$, so the Nernst heat theorem is satisfied. Moreover, the entropy approaches zero from the positive side.

\section{Discussion}

The zero contribution of s polarization to the $n=0$ term has solid physical grounds. In the local case the $1 / \omega$ behaviour of the dielectric function, responsible for the vanishing of the reflection coefficient $r_{s}$, is the direct result of Ohm's law. Our analysis revealed that discontinuity between real and ideal metals appears due to contribution of the evanescent fields for real metals. This contribution depends only on the existence of the relaxation frequency but not on its value. Even an infinitely small $\omega_{\tau}$ will result in zero contribution of s polarization. When $\omega_{\tau} \rightarrow 0$ the Drude model turns to the plasma model. Therefore, one must use correctly the plasma model: $\omega_{\tau}$ can be infinitely small but finite. It is similar to the situation with the free-space propagator when infinitely small absorption of vacuum is important for right definition of the propagator. Nonzero $\omega_{\tau}$ is also important for the dispersion relation in the plasma model.

There is an additional very simple physical explanation why s polarization should not contribute to the force in the low-frequency limit. If $z$ is the normal direction to the metal 
surface, then s-polarized field can be chosen as having the following nonzero components of magnetic and electric fields: $H_{x}, H_{z}$ and $E_{y}$. When $\omega \rightarrow 0$ the magnetic field can be found from the Maxwell equation $\nabla \times \mathbf{H}=4 \pi \mathbf{j} / c$, where $\mathbf{j}$ is the external current density responsible for the fluctuating fields [16]. The electric field, which is described by the equation $\nabla \times \mathbf{E}=\mathrm{i} \omega \mathbf{H} / c$, will be suppressed in comparison with $\mathbf{H}$ because $\omega$ is small. So in the limit $\omega \rightarrow 0$ s-polarized field degenerates to a pure magnetic field. But the magnetic field penetrates freely via nonmagnetic metals which means that the reflection coefficient is going to zero. Similarly the $p$-polarized field degenerates to a pure electric field in the $\omega \rightarrow 0$ limit. The electric field is screened by the metal and the reflection coefficient is 1 .

The low-temperature behaviour of the Casimir entropy is an additional point of controversy. It was argued [23] that $S$ is going to zero with $T$ only if the plasma model is used for the $n=0$ term. We stressed $[25,27]$ that to analyse the low-temperature behaviour one has to take into consideration the anomalous skin effect. Description of nonlocal metals can be done with two nonlocal dielectric functions [26] or equivalently with two impedances [30], which are known in the theory of metals. It was demonstrated that the Leontovich impedance, which is used for the description of propagating fields, cannot be applied for the Casimir problem because evanescent fields are important in this case. The relaxation frequency decreases fast with $T$ but its role takes the nonlocal frequency $v_{F} k$. It was shown numerically [26] and analytically [27] that the entropy disappears with $T$. In contrast, for the plasma model the entropy will stay constant at $T=0$. In this paper we refined our previous analytical result and showed that at very low-temperatures $S \sim T$.

Although there is a physical understanding why s polarization does not contribute to the $n=0$ term, the experimental situation, to all appearance, is not in its favour. Absence of $\mathrm{s}$ polarization contradicts to Lamoreaux experiment [4]. Recently Decca et al [10] refined their measurements reducing the surface roughness and increasing precision of determination of the absolute separation. They did not measure the optical properties of the used gold films and for this reason the theoretical force was, probably, overestimated. Nevertheless, even with the use of the handbook optical data one can conclude that absence of s polarization, probably, is not supported by this experiment. Therefore, the thermal Casimir problem is still an open question.

\section{References}

[1] Casimir H B G 1949 Proc. K. Ned. Akad. Wet. 51793

[2] Milonni P W 1994 The Quantum Vacuum (San Diego, CA: Academic)

[3] Elizalde E, Odintsov S D, Romeo A, Bytsenko A A and Zerbini S 1994 Zeta Regularization with Applications (Singapore: World Scientific)

[4] Lamoreaux S K 1997 Phys. Rev. Lett. 785

Lamoreaux S K 1998 Phys. Rev. Lett. 815475

[5] Mohideen U and Roy A 1998 Phys. Rev. Lett. 814549

Roy A, Lin C-Y and Mohideen U 1999 Phys. Rev. D $60111101(\mathrm{R})$

Harris B W, Chen F and Mohideen U 2000 Phys. Rev. A 62052109

[6] Ederth T 2000 Phys. Rev. A 62062104

[7] Chan H B, Aksyuk V A, Kleiman R N, Bishop D J and Capasso F 2001 Science 2911941

Chan H B, Aksyuk V A, Kleiman R N, Bishop D J and Capasso F 2001 Phys. Rev. Lett. 87211801

[8] Bressi G, Carugno G, Onofrio R and Ruoso G 2002 Phys. Rev. Lett. 88041804

[9] Decca R S, López D, Fischbach E and Krause D E 2003 Phys. Rev. Lett. 91050402

Decca R S, Fischbach E, Klimchitskaya G L, Krause D E, López D and Mostepanenko V M 2003 Phys. Rev. D 68116003

[10] Decca R S, López D, Fischbach E, Klimchitskaya G L, Krause D E and Mostepanenko V M 2005 Ann. Phys. 31837 
[11] Iannuzzi D, Lisanti M and Capasso F 2004 Proc. Natl Acad. Sci. USA 1014019

Lissanti M, Iannuzzi D and Capasso F 2005 Proc. Natl Acad. Sci. USA 10211989

[12] Milton K A 2004 J. Phys. A: Math. Gen. 37 R209

[13] Mehra J 1967 Physica 37145

[14] Brown L S and Maclay G J 1969 Phys. Rev. 1841272

[15] Lifshitz E M 1956 Zh. Eksp. Teor. Fiz. 2994

Lifshitz E M 1956 Sov. Phys._JETP 273

[16] Lifshitz E M and Pitaevskii L P 1980 Statistical Physics part 2 (Oxford: Pergamon) (Engl. Transl.)

[17] Schwinger J, DeRaad L L and Milton K A 1978 Ann. Phys., NY 1151

[18] Boström M and Sernelius B E 2000 Phys. Rev. Lett. 844757

[19] Bordag M, Geyer B, Klimchitskaya G L and Mostepanenko V M 2000 Phys. Rev. Lett. 85503

[20] Svetovoy V B and Lokhanin M V 2000 Mod. Phys. Lett. A 151013

Svetovoy V B and Lokhanin M V 2000 Mod. Phys. Lett. A 151437

[21] Svetovoy V B and Lokhanin M V 2001 Phys. Lett. A 280177

[22] Høye J S, Brevik I, Aarseth J B and Milton K A 2003 Phys. Rev. E 67056116

[23] Bezerra V B, Klimchitskaya G L and Mostepanenko V M 2002 Phys. Rev. A 65052113

[24] Boström M and Sernelius B E 2004 Physica A 33953

[25] Svetovoy V B and Lokhanin M V 2003 Phys. Rev. A 67022113

[26] Sernelius Bo E 2005 Phys. Rev. B 71235114

[27] Svetovoy V B and Esquivel R 2005 Phys. Rev. E 72036113

[28] Torgerson J R and Lamoreaux S K 2004 Phys. Rev. E 70047102

[29] Kliewer K L and Fuchs R 1968 Phys. Rev. 172607

[30] Esquivel R and Svetovoy V B 2004 Phys. Rev. A 69062102

[31] Mochán W L, Villarreal C and Esquivel-Sirvent R 2002 Rev. Mex. Fis. 48339

[32] Esquivel R, Villarreal C and Mochán W L 2003 Phys. Rev. A 68052103

[33] Abrikosov A A 1988 Fundamentals of the Theory of Metals (Amsterdam: North-Holland)

[34] Geyer B, Klimchitskaya G L and Mostepanenko V M 2003 Phys. Rev. A 67062102 